Ann. Biol. anim., Bioch., Biophys., I965, 5 (4), 527-533.

\title{
ENREGISTREMENT DE L'ACTIVITÉ UNITAIRE DES AFFÉRENCES VAGALES CHEZ LES PETITS RUMINANTS ESSAI DE RÉCEPTION PAR MICROELECTRODES AU NIVEAU DU GANGLION PLEXIFORME
}

\author{
J. P. ROUSSEAU \\ Département de Neurophysiologie végétative de l'Institut de Neurophysiologie et Psychophysiologie, \\ Marseille (Bouches-du-Rhône) \\ Centre national de Recherches zootechniques, Département de Physiologie animale, \\ Jouy-en-Josas (Seine-et-Oise)
}

SOMMAIRE,

L'activité de 28 neurones sensitifs du vague a été recueillie au niveau du ganglion plexiforme chez 26 petits ruminants (boucs et agneaux) à l'aide de microélectrodes extra cellulaires.

Ces afférences étaient surtout d'origine respiratoire, mais également d'origine cardiaque, œsophagienne et gastrique.

Nous nous sommes heurté à d'importantes difficultés en partie dues à la richesse du ganglion en un tissu conjonctif, dense et résistant. Nous pensons que cette technique de réception, pleinement réalisable chez le Chat, ne peut être fructueuse chez les Ruminants qu'à condition d'utiliser des animaux très jeunes.

La technique de la "fibre isolée " a permis de révéler l'existence de divers types de récepteurs au niveau des viscères thoraciques et abdominaux (ADRIAN, I933; WhitTERIDGE, I948; PAINTAL, I952, I953, I954, I963; IGGO, I955, I957, I958). I, a plupart de ces résultats ont été obtenus chez le Chat et, à notre connaissance, il n'existe aucune publication relative à l'activité des récepteurs pulmonaires, cardiaques ou œesophagiens des Ruminants. Seuls les récepteurs gastriques ont été mis en évidence chez ces animaux par IGGo (I955), qui a étudié II fibres, et par RuckeвUSCH (1963), qui a recueilli des décharges vagales synchrones de la contraction réticulaire spontanée. Nous nous sommes demandé si l'enregistrement par microélectrodes de l'activité des cellules du ganglion plexiforme, technique mise au point par MEI ( 1962 ) pour étudier les afférences vagales du Chat, ne pourrait pas se révéler 
commode également chez les petits Ruminants. Nous nous sommes heurté à de grosses difficultés, mais compte tenu de la rareté des données bibliographiques, nous croyons utile de rapporter nos résultats.

\section{TECHNIQUES}

Nos expériences portent sur 17 boucs âgés de 7 à I 2 mois et sur 9 agneaux de 45 à Ioo jours.

L'animal est anesthésié au Nembutal. Le ganglion plexiforme est découvert selon la technique décrite par DusSardier ( tive, il est placé sur un support anti-vibratoire qui le protège partiellement des mouvements parasites. Pour recueillir l'activité des cellules ganglionnaires, nous utilisons des micropipettes de verre remplies de $\mathrm{KCl}_{3} \mathrm{M}$, dont le diamètre à la pointe est de $\mathrm{I}$ à $2 \mu$. Nous enregistrons également l'électrocardiogramme, le pneumogramme et les contractions cesophagiennes ou gastriques obtenues ra sstimulation de l'extrémité périphérique du vague droit sectionné.

\section{RÉSULTATS. -- DISCUSSION}

\section{Valeur de la technique utilisée}

Cette technique de réception par microélectrodes, pleinement réalisable chez le Chat, n'est pas aussi fructueuse chez les petits Ruminants. S'il est relativement facile, par des artifices expérimentaux, de réduire l'amplitude des mouvements produits par le pouls carotidien et la respiration, on ne peut pas les faire disparaitre suffisamment pour conserver l'activité d'un neurone pendant le temps nécessaire pour localiser le récepteur ou agir sur lui. La présence d'un tissu conjonctif abondant et dur limite également la qualité et la quantité des enregistrements. La pointe des micropipettes de verre casse rapidement : elle n'est plus alors assez sélective pour recueillir l'activité d'un seul neurone, ou bien elle lèse définitivement la celule dont l'état de souffrance se traduit par une décharge de fréquence élevée. Le shunt électrique réalisé par des ponts de tissu conjonctif restreint par ailleurs l'amplitude des phénomènes qui émergent à peine du bruit de fond du système électronique. L'emploi de la hyaluronidase n'améliore pas nos enregistrements, car cet enzyme, qui ramollit la texture dense et fibreuse du ganglion, n'a pas d'effet sur la capsule des cellules nobles. Ces difficultés expliquent le petit nombre de neurones dont nous avons enregistré les activités (28). Encore faut-il noter que nous avons réussi à en étudier I7 chez 9 agneaux, mais seulement II chez I7 boucs.

\section{Discussion.}

Devant les écueils rencontrés, inhérents surtout à la constitution anatomique du ganglion, il apparaît difficile d'utiliser couramment sur des boucs adultes la technique envisagée. Les résultats sont moins décevants lorsqu'on travaille sur des agneaux dont le tissu conjonctif ganglionnaire, moins dense, permet une pénétration plus aisée des micropipettes. Il apparaît certain que ces animaux constituent un matériel meilleur que le Bouc adulte pour ce type de recherches. 


\section{Récepteurs respiratoires}

Nous avons observé l'activité de 20 neurones respiratoires.

Onze d'entre eux présentaient une activité permanente. Leur rythme de base variait d'un neurone à l'autre dans des limites assez larges (de I6 à 45 impulsions par seconde). En respiration spontanée, la fréquence de décharge d'un même neurone augmentait au cours de 1'inspiration (fig. IA). Elle s'élevait également lorsqu'en respiration artificielle on accroissait le volume d'air inspiré (fig. 2).

Huit récepteurs pulsaient seulement au cours de l'inspiration (fig. IB) ; les uns montraient une activité dès le début de l'inspiration, et la fréquence de leur. décharge augmentait progressivement pour se maintenir à un niveaut relativement constant (8 à Io/s) pendant toute la durée de l'inflation des poumons (fig. 3). Les autres se manifestaient à la fin de la phase inspiratoire par une courte décharge de quelques potentiels dont la fréquence $(25 / \mathrm{s})$ était beaucoup plus élevée que celle des récepteurs précédents (fig. IC) ; chez 1'Agneau ventilé artificiellement, le nombre et la fréquence des influx constituant la salve augmentaient lorsqu'on faisait varier de $\mathrm{I} 50$ à $200 \mathrm{ml}$ le volume de l'air inspiré.

Enfin, un récepteur était actif à deux moments du cycle respiratoire : à la fin de l'expiration et au sommet de l'inspiration. Dans ce cas, la réponse à la déflation était toujours plus variable que la réponse inspiratoire (fig. ID).

\section{Discussion.}

Nous avons retrouvé chez les petits Ruminants les divers types de récepteurs respiratoires déjà connus. Los activités permanentes apparaissent relativement plus nombreuses que chaz le Chat (MEI, I952). Celles présentant de courtes décharges au sommet de l'inspiration sont obtenues principalement lorsque l'animal est placé sous respiration artificielle. L'augmentation du volume d'air inspiré fait croître leur fréquence et, de plus, fait décharger des récepteurs jusqu'ici silencieux. Il s'agit vraisemblablement dans les deux cas de récepteurs à adaption rapide.

\section{Récepteurs cardiaques}

Quatre récepteurs présentaient des périodes d'activité se situant après la fin du complexe QRS, ou bien au cours de l'onde T de l'électrocardiogramme. Deux d'entre eux étaient des barorécepteurs artériels. Leur volée d'influx, de latence courte par rapport au début de l'onde $Q$ de 1 'ECG (58 et I $23 \mathrm{~ms}$ ), se poursuivait au-delà de la moitié de la systole ventriculaire. Le pic de fréquence de 70 à 80 influx par seconde, immédiatement atteint, décroissait ensuite très rapidement (fig. 4).

Un troisième récepteur répondait aux critères d'une terminaison atriale du type B. La décharge se situait tardivement au cours de la systole ventriculaire: $2 \mathrm{r} 4 \mathrm{~ms}$ après l'onde $Q$; sa fréquence augmentait graduellement de $48 / \mathrm{s}$ à $67 / \mathrm{s}$, puis tombait rapidement à sa valeur initiale à la fin de la révolution cardiaque. Le nom. bre d'influx de chaque volée variait périodiquement avec la respiration.

Nous avons également enregistré l'activité d'un neurone qui, se produisant juste après 1'onde $Q\left(4^{8} \mathrm{~ms}\right)$, persistait pendant toute la durée de la systole ventriculaire et de la phase isoélectrique de l'électrocardiogramme. La fréquence de dé-

Annales de Biologie animale. - 1965 . 


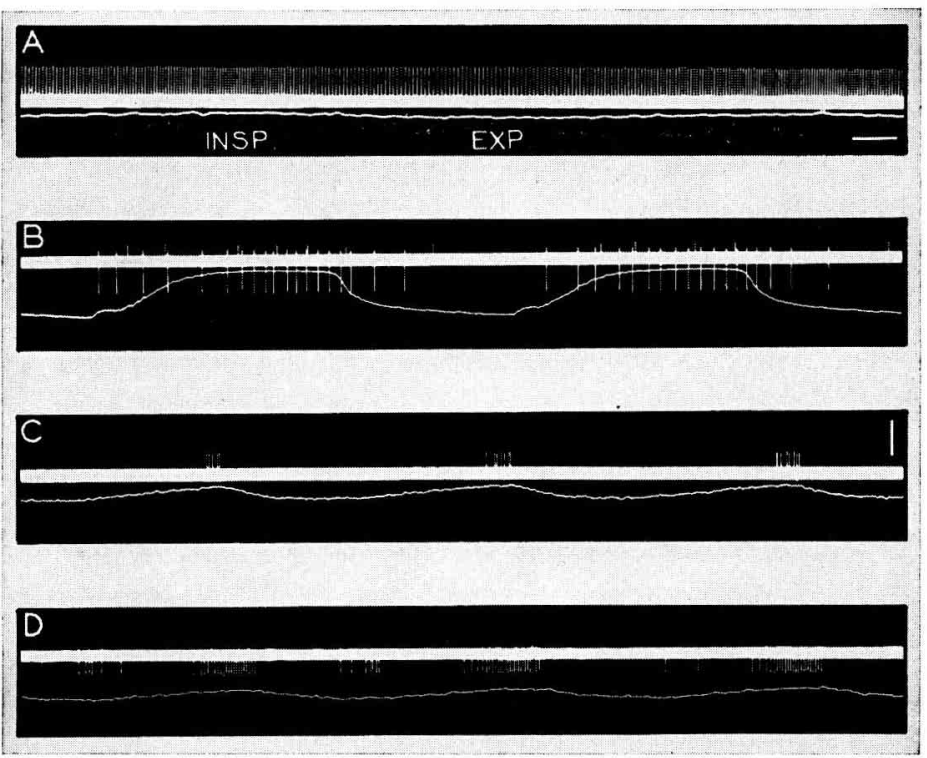

FIG. I. -- Différents types de récepteurs respiraloires

A - Récepteur pulsant en permanence. La fréquence de sa décharge augmente au cours de l'inspiration.

B - Récepteur pulsant pendant toute la durée de l'inspiration et une partie de l'expiration. ratoire.

C - Récepteur se manifestant seulement par une courte décharge au sommet de chaque phase inspi-

D - Récepteur actif à deux moments du cycle respiratoire : à la fin de l'expiration et au sommet de l'inspiration.

Étalonnage pour $\mathrm{A}: 0,5 \mathrm{mV}-0,3 \mathrm{~s}$; - B, C et D: $0,5 \mathrm{mV}-0,5 \mathrm{~s}$.

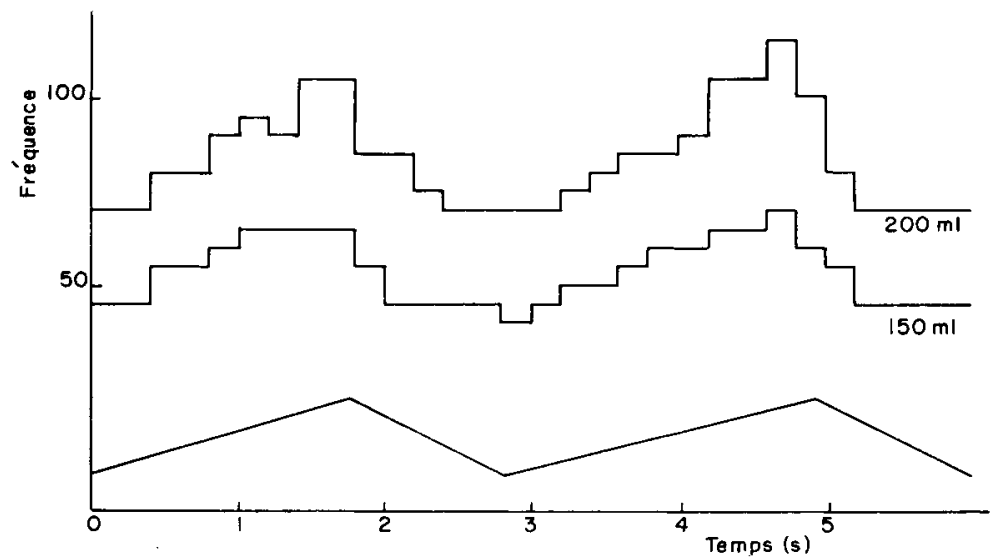

FIG. 2. - Décharge d'un récepteur respiratoire pulsant en permanence

Évolution de la fréquence en fonction du volume de l'air inspiré.

La fréquence augmente au cours de chaque inspiration, pour un volume d'air courant donné. Elle s'accroit également lorsqu'on fait varier de 150 à $200 \mathrm{ml}$ le volume d'air inspiré.

Les fréquences ont été comptées par tranches de $200 \mathrm{~ms}$.

$\mathrm{La}$ ligne inférieure représente le pneumogramme, l'inspiration correspondant à une déflexion vers le huut du tracé. 


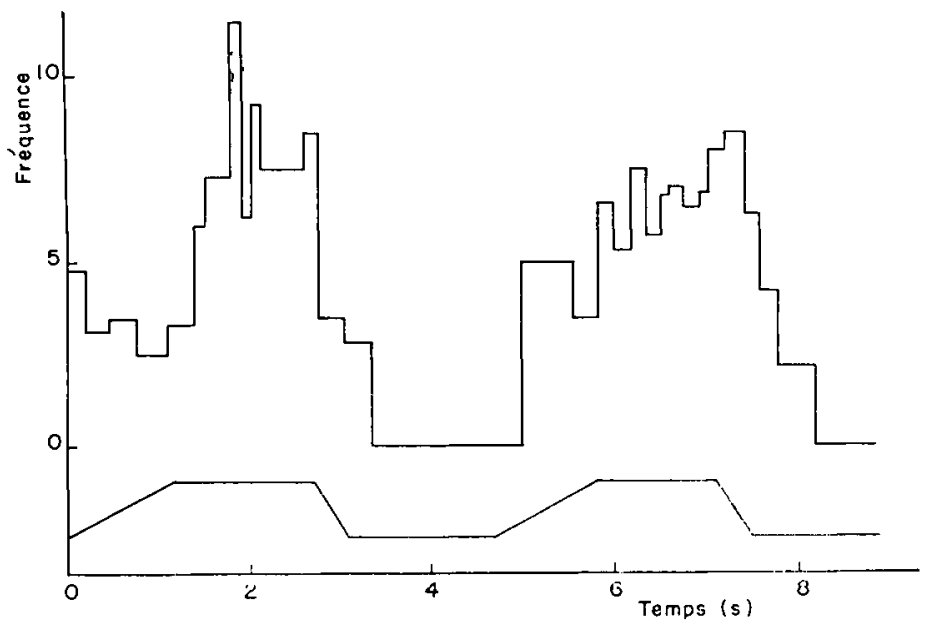

FIG. 3. - Augmentalion, au cours de l'inspiration, de la tréquence instantanée de décharge d'un récepteur respiratoire excité seulement par l'inflation des poumons

La ligne inférieure représente le pneumogramme l'inspiration correspondant à une déflexion vers le haut du tracé.

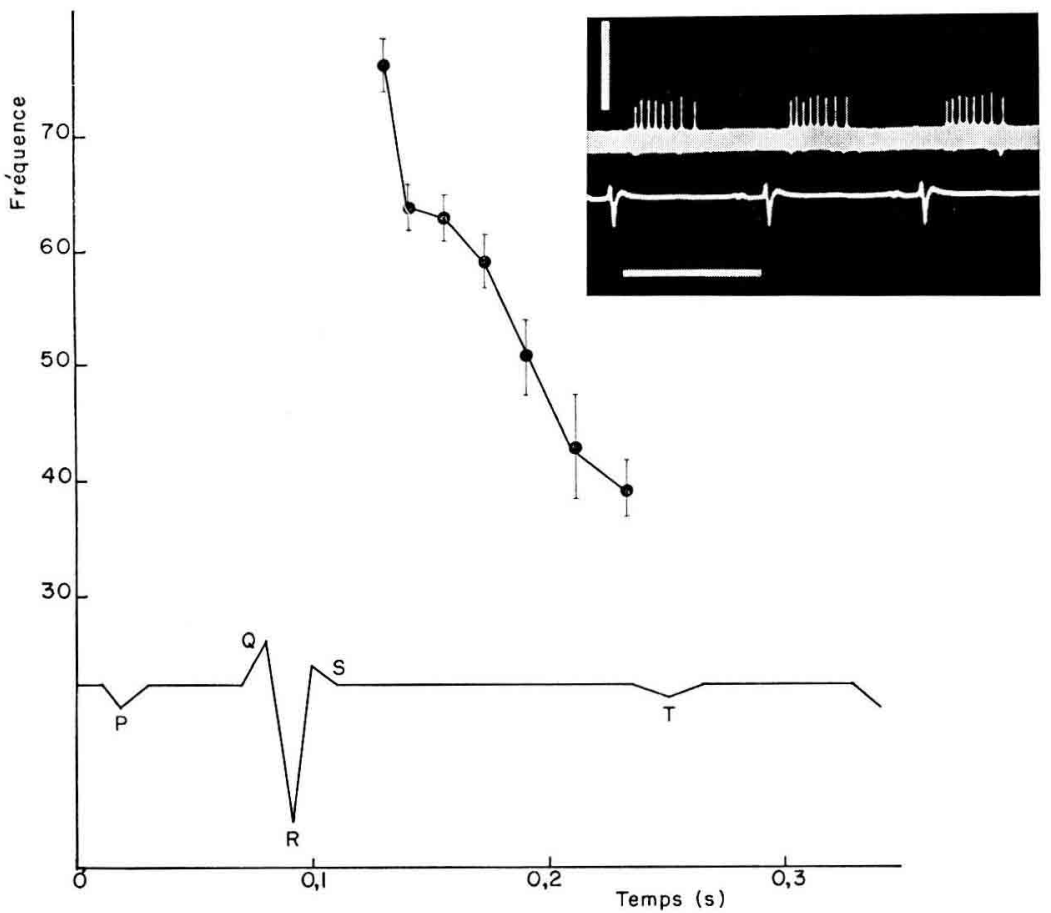

FIG. 4. - Étude de la décharge d'un barorécepteur artériel

Ce récepteur pulse très tôt par rapport à l'onde $Q$ de l'E.C.G. Sa décharge se poursuit au-delà de la moitié de la systole ventriculaire. Ia fréquence maximum immédiatement atteinte décroît brutalement en une centaine de millisecondes. Etalonnage $: 0,5 \mathrm{mV}-0,3 \mathrm{~s}$. 
charge se maintenait pendant près de $\mathrm{I} 50 \mathrm{~ms}$ à un niveau moyen de $\mathrm{I} 9$ influx par seconde, puis suivait une variation en crescendo identique à celle signalée précédemment.

\section{Discussion.}

Ces résultats sont à rapprocher de ceux déjà obtenus par d'autres auteurs chez le Chat, qu'ils aient trait aux barorécepteurs étıdiés par HEYMANs et NEIL (I958) ou aux terminaisons atriales du type $\mathrm{B}$ décrites par WHIT'TERIDGE (I948) et PAINTAI, (I953). Cependant, un seul neurone est difficile à cataloguer : il pulsait très précocément après l'onde $Q$ de l'électrocardiogramme, ce qui pouvait faire penser à un récepteur ventriculaire, mais sa décharge se prolongeait bien au-delà de l'onde $T$. Il s'agit peut-être d'un récepteur du tỵpe $B$ faiblement sollicité par un retour pathologique du sang vers l'oreillette juste après la mise en tension du ventricule, puis normalement activé par la réplétion plus tardive de la cavité auriculaire.

\section{Récepteurs asophagien et gastrique}

Nous avons placé dans la lumière de l'osophage thoracique un ballonnet, rempli de $20 \mathrm{ml}$ d'air. La distension passive ainsi réalisée mettait en jeu un récepteur asophagien dont l'activité persistait tant qu'était maintenue la distension.

Ia faradisation de l'extrémité périphérique du nerf vague droit nous a permis d'étudier trois netrones sensitifs ayant une activité synchrone de la contraction réticulaire ainsi produite. Un seul, dans nos conditions expérimentales, pulsait irrégulièrement, en dehors de toute stimulation, en bouffées de 4 à 50 potentiels dont la fréquence variait de 8 à 30 par seconde, sans qu'apparût un changement dans la pression intragastrique.

\section{Discussion.}

Ces résultats sont trop pau nombreux pour nous permettre d'entımer une discussion. Il faut cependant noter que la fréquence de décharge des récepteurs rencontrés ne suit pas les variations de la pression intragastrique. Ie stimulus ne semble donc pas être l'augmentation de la pression, mais plutôt la variation de la tension des fibres musculaires lisses dont le système d'enregistrement utilisé ne peut rendre compte d'une façon satisfaisante. On peut aussi, comme IGGo (I955), rapporter les décharges intermittentes que nous avons rencontrées en dehors de toute modification de la pression, à la stimulation du récepteur lors d'une contraction locale intrinsèque de l'estomac.

Reçu pour publication en juillet 1965 .

\section{SUMMARY}

RECORDING OF SINGLE UNIT ACTIVITY OF AFFLRENT VAGAL FIBRES IN TIIE SMALI, RUMINANTS. TRIAL WITH MICROELECTRODES AT TIIE LETEL, OF TILE NODOSE GANGLION

The activity of 28 sensory neurons of the vagus nerve has been recorded at the level of the nodose ganglion in 26 small Ruminants (goats and lambs) with extracellular microelectrodes. 
These afferent units were mostly of respiratory origin, but also of cardiac, œesophageal and gastric origin. We met great difficulties due to the large amount of dense connective tissue in the ganglion. We think that this recording technique, quite possible in the Cat, can be successful in the Ruminants only when using very young animals.

\section{RÉFÉRENCES BIBLIOGRAPHIQUES}

Adrian E. D., 1933. Afferent impulses in the vagus and their effect on respiration. J. Physiol., London, 79, $33^{2-3}-35^{8}$

Dussardier M., ig60. Recherches sur le contrôle bulbaire de la motricilé gaslrique chez les Ruminants. Thèse Doctorat ès-Sciences, Paris, I 96 p. I.N.R.A. édit.

Heymans C., Neit E., I958. Reflexogenic areas of the cardiovascular system. London, J. and A. Churchill Ltd., 27 I p.

IGGo A., I955. Tension receptors in the stomach and the urinary bladder. J. Physiol., London, 128, 593607 .

IGGo A., I957. Gastrointestinal tension receptors with unmyelinated afferent fibres in the vagus of the Cat. Quart. J. exper. Physiol., 42, I 30-143.

IGGO A., I958. The electrophysiological identification of single nerve fibres with particular reference to the lowest conducting vagal afferent fibres in the Cat. J. Physiol., London, 142, I Io- 26.

MeI N., I962. Enregistrement de l'activité unitaire des afférences vagales. Réception par microélectrodes au niveau du ganglion plexiforme. Ann. Biol. anim. Bioch. Biopliys., 2, 361-364.

Paintal A. S., I952. Conduction velocity of single respiratory and cardiovascular afferent fibres in the cervical vagus. J. Physiol., London, 117, to P.

Paivial A. S., I953. A study of rioht and left atrial receptors. J. Plystol., London, 120, 596-6ro.

Paintal A. S., I954. A study of gastric suetch receptors. Their role in the peripheral mechanism of satiation of hunger and thirst. J. Physiol., london, 126, $255^{+270 .}$

Paintal A. S., r963. Vagal afferent fibres Ergebn. Physiol., 52, 75-156.

RUCKEBUSCH Y., I963. Recherches sur la rigulation centrale du comporlement alimentaire chez les Ruminants Thèse Doctorat ès-Sciences, Lyon, $213 \mathrm{p}$.

WiItTERIDGe D., 1948. Afferent nerve fibres from the heart and lungs in the cervical vagus. J. Physiof. London, 10\%, 496-512. 